J Am Acad Dermatol. 2009 July ; 61(1): 66-72. doi:10.1016/j.jaad.2009.02.011.

\title{
Statin Use and Risk of Basal Cell Carcinoma
}

\author{
Maryam M. Asgari, MD MPH ${ }^{1,2}$, Jean Tang, MD PhD2,4, Ervin H. Epstein Jr., MD ${ }^{4}$, Mary- \\ Margaret Chren, MD ${ }^{2}, 3$, E. Margaret Warton, MPH $^{1}$, Charles P. Quesenberry Jr., PhD ${ }^{1}$, Alan \\ S. Go, MD ${ }^{1,5}$, and Gary D. Friedman, MD MS ${ }^{1,6}$ \\ 1Division of Research, Kaiser Permanente Northern California, Oakland, California
}

2Department of Dermatology, University of California at San Francisco, San Francisco, California, San Francisco, California

3 Health Services Research Enhancement Award Program (REAP), San Francisco Veterans Affairs Medical Center, San Francisco, California

4Children's Hospital Oakland Research Institute, Oakland, California

5Departments of Epidemiology, Biostatistics and Medicine, University of California, San Francisco, San Francisco, California

6Department of Health Research and Policy, Stanford University School of Medicine, Stanford, California

\section{Abstract}

OBJECTIVE-We examined the association between statin use and basal cell carcinoma (BCC) risk.

\begin{abstract}
METHODS-We identified all members of a large integrated healthcare delivery system diagnosed with a histologically-proven BCC in 1997. Subsequent BCCs were identified through 2006 from health plan electronic pathology records. Longitudinal exposure to statins and other lipid-lowering agents was determined from automated pharmacy records. We used extended Cox regression to examine the independent association between receipt of statin therapy (ever vs. never, cumulative duration) and risk of subsequent BCC. To minimize confounding by indication, we conducted sensitivity analyses in the subset of individuals considered eligible for lipid-lowering therapy based on national guidelines.
\end{abstract}

RESULTS-Among 12,123 members diagnosed with BCC who had no prior statin exposure, 6381 developed a subsequent BCC during follow-up. Neither ever use of statins (adjusted hazard ratio [aHR] 1.02, 95\% CI: 0.92-1.12) or cumulative duration of statin (aHR 1.02 per year, 95\% CI: 0.99-1.11) was associated with subsequent BCC after adjustment for age, sex, and healthcare utilization. Risk estimates did not change appreciably when the analysis was limited to the subset of individuals who met eligibility criteria for initiating statin therapy. There was also no significant

Corresponding Author: Maryam Asgari, MD MPH, Kaiser Permanente Northern California, Division of Research, 2000 Broadway, Oakland, CA 94612, (510)891-3895 (phone), (510)891-3606 (fax), E-mail: E-mail: maryam.m.asgari@kp.org.

Conflict of Interest Disclosure: Dr. Friedman served on an advisory committee for Roche Pharmaceuticals in June 2008. He has consulted for law firms serving the plaintiffs regarding litigation concerning celecoxib and serving Ortho-McNeil-Janssen Pharmaceuticals regarding litigation concerning Ortho-Evra. None of these associations influenced his work on this paper. The remaining authors have no conflict of interest to disclose.

Publisher's Disclaimer: This is a PDF file of an unedited manuscript that has been accepted for publication. As a service to our customers we are providing this early version of the manuscript. The manuscript will undergo copyediting, typesetting, and review of the resulting proof before it is published in its final citable form. Please note that during the production process errors may be discovered which could affect the content, and all legal disclaimers that apply to the journal pertain. 
association between use of non-statin antilipemics and subsequent BCC (aHR 1.10, 95\% CI: 0.76-1.58).

LIMITATIONS—No information was available for BCC risk factors, such as sun sensitivity and sun exposure.

CONCLUSIONS-Among a large cohort of individuals with BCC, statin therapy was not significantly associated with risk of subsequent BCC.

\section{INTRODUCTION}

Basal cell carcinoma (BCC) is the most common cancer in the U.S., affecting nearly one million Americans annually ${ }^{1}$ and its incidence is rising. 2,3 Although BCCs are rarely fatal, their high incidence and the frequent occurrence of new primary BCCs in affected individuals ${ }^{4}$ can cause significant morbidity. BCCs also pose a substantial financial impact and are among the most costly cancers to treat in the Medicare population. ${ }^{5}$ The primary known risk factors for BCC are sun sensitivity and exposure to ultraviolet (UV) radiation. To date, no effective, welltolerated chemopreventive agents exist for BCC. Such therapies would be especially useful for individuals with a prior BCC given that nearly $44 \%$ go on to develop a subsequent BCC over a 3 -year follow-up period. ${ }^{6}$

HMG-CoA reductase inhibitors (statins) are lipid-lowering drugs that effectively reduce cardiovascular risk and are the most commonly prescribed medication in the U.S. ${ }^{7}$ Statins have also been explored for other potentially beneficial health effects, including chemoprevention. Laboratory data support the possible role of statins for the chemoprevention of BCCs. Statininduced cholesterol depletion induces apoptosis of cultured keratinocyte cell lines. ${ }^{8}$ They also inhibit the sonic hedgehog signaling pathway ${ }^{9-10}$ which is the pivotal molecular pathway in BCC carcinogenesis. ${ }^{11}$ Statins have also been shown to have potent inhibitory effects on cancer cells in vitro and in animal models, $12-13$ but clinical studies on cancers other than skin cancer have reached mixed conclusions. ${ }^{14-17}$ Based on these laboratory and epidemiologic data, we hypothesized that statin use would be associated with a reduction in risk of BCCs in a population at high-risk for their development. We tested this hypothesis in a large integrated healthcare delivery system by examining the relation between statin therapy and risk of subsequent BCC among a large cohort of Kaiser Permanente of Northern California (KPNC) members previously diagnosed with BCC.

\section{METHODS}

We conducted a longitudinal cohort study of all KPNC members diagnosed with a BCC in 1997 to examine the association between statin exposure and subsequent BCC risk. In secondary analysis, we limited the cohort to those members who were eligible for statin treatment to minimize confounding by indication. Finally, we examined exposure to non-statin antilipemics and risk of subsequent BCC using the full cohort to assess whether any associations were statin-specific or related to the lowering of cholesterol levels.

\section{Study Population}

We identified all KPNC members with a histologically proven $\mathrm{BCC}$ from electronic health plan pathology records with an assigned SNOMED code of 809XX - 811XX (with the exception of 81000 and 81100), 80003, or 80103 and who also had a pathology report that contained the text-string "basal cell carcinoma" or "BCC" in the diagnosis line and the topography code of "skin." In 1997, we identified 18,305 BCCs diagnosed in 13,063 individuals. We excluded individuals who had medical record numbers that could not be matched to their membership file or had missing key membership information $(n=55)$ and members who did not have active status at the time of BCC diagnosis ( $n=98)$, leaving 12,910 
individuals for analysis. This study was approved by the Kaiser Foundation Research Institute Institutional Review Board.

\section{Exposure to Statin Therapy}

Longitudinal exposure to statin therapy was ascertained for each cohort member using information found in automated KPNC pharmacy databases on filled prescriptions between the index BCC diagnosis date through December 2006. We searched for all known available statins during the study period: lovastatin, simvastatin, atorvastatin, pravastatin, fluvastatin, and cerivastatin. We assigned periods of continuous statin exposure during follow-up for all cohort members using data from serial prescriptions based on each prescription's fill date, days supply of pills dispensed, and estimated days supply. ${ }^{18}$ For any two consecutive prescriptions, we considered an individual as continuously receiving statins if the gap between the expected end date of the first prescription and fill date of the second prescription was $\leq 30$ days. If the gap was >30 days, the individual was considered off statins until the fill date of the next prescription. Individuals who had any documented continuous statin exposure before the index date were excluded from all models $(n=787)$.

\section{Follow-up and Outcome}

Subjects were followed from the date of their first diagnosis in 1997 until any of the following occurred: subsequent BCC, disenrollment from the health plan membership defined as a gap of more than 90 days, death, or the end of the study period on December 31, 2006. The primary outcome was subsequent BCC identified from health plan pathology records screened up to each subject's censoring date using the same SNOMED codes defined for study entry.

\section{Covariates}

Age and sex were ascertained from administrative databases. Because we hypothesized that individuals receiving statins were more likely to use the healthcare system overall and more likely to visit a dermatologist who would biopsy their tumor, we assessed health care utilization to account for possible screening bias. Health care utilization was defined as an annualized rate of medical outpatient and emergency room visits during the follow-up period. Annualized dermatology-specific healthcare utilization was also ascertained, with the assumption that dermatology visits would be where biopsies of BCCs were most likely to occur. However, results did not change when adjusting for dermatology-specific resource utilization, so only results that accounted for differences in overall health care utilization are described.

\section{Statistical Analysis}

We examined the potential impact of statins on subsequent BCC risk with two different measures of statin exposure using an extended Cox regression to account for time-dependent variables and immortal time bias. ${ }^{18}$ First, we used an "ever vs. never use" approach in which we assigned persons who initiated statin therapy as being continuously on statins through the follow-up period starting with the first prescription lasting at least 30 days filled on or after index date and evidence of at least one refill. If an individual initially filled only a single prescription but no refills, we assumed that the individual had not taken the pills as prescribed due to problems with side effects or other reasons, and these individuals were considered unexposed $(n=31)$. Second, to investigate the possible effect of duration of exposure on subsequent BCC, we calculated the cumulative days supply of statin therapy during followup. Cumulative exposure was calculated by determining the date on which each exposed cohort member reached an additional year of statin exposure. Cumulative exposure was also examined by categories: unexposed, short-term ( 1 day-2 years), moderate ( $>2-5$ years), and long-term ( $>5$ years) use. In addition, we performed separate sensitivity analyses varying the lag time before exposure to statins would be considered to have an impact on BCC formation ( 0 or 90 
days), as well as including cases of very early occurrence of subsequent BCC ( $\leq 90$ days after index date), and late occurrences (over 180 days and over 365 days after index date).

To assess whether the exposure was statin-specific or related to the lowering of cholesterol levels by other pharmacological methods, we also examined exposure to non-statin antilipemics. Given the limited number of cohort members who took these agents in the absence of concomitant statin therapy, we only used an "ever vs. never use" approach. In addition, for this analysis, we excluded observation time for any individual after the date they began use of statins.

Finally, to further address the possibility of residual confounding by indication, we identified the subset of cohort members who were eligible for lipid-lowering therapy based on national clinical practice guidelines ${ }^{20}$ during the study period and who did not have prior known statin therapy. We repeated all analyses in this subset for comparison with the main results.

\section{RESULTS \\ Cohort Characteristics}

Among 12,910 individuals diagnosed with BCC, 787 had known statin exposure at cohort entry and were excluded, leaving 12,123 subjects for analysis. Mean age was $64.7 \pm 14.1$ years and $58.9 \%$ were men. Of the 12,123 subjects, 3309 (27.3\%) were lost to follow-up during the 10 year period. The mean age of those members who were lost to follow-up (66.03 years) and their gender distribution ( $58.4 \%$ male) did not substantially differ from the demographics of the full cohort. During follow-up, 1,535 subjects received statin therapy, and there was no significant difference in gender $(59.1 \%$ male exposed vs. $58.8 \%$ male unexposed, $\mathrm{p}=0.85)$. The mean number of years of follow-up was 4.24 years (median 3.14 years). Mean age at entry was slightly but significantly $(\mathrm{p}=0.01)$ higher in the stain-exposed (65.3 years) than in the statinunexposed (64.6 years) patients. The median number of statin prescriptions filled per treated individual during follow-up was 10 (interquartile range: 5-18) with median 828 days of exposure (interquartile range 392-1460). The most commonly prescribed statins were lovastatin $(75.2 \%)$, simvastatin (19.9\%), atorvastatin (3.9\%), pravastatin $(0.8 \%)$, simvastatin/ ezetimibe $(0.1 \%)$ and fluvastatin $(0.1 \%)$.

Overall, only 120 cohort members received non-statin antilipemics, which included gemfibrozil (47.1\%) niacin (26.0\%), cholestyramine (13.6\%), colestipol (6.3\%), ezetimibe $(4.2 \%)$, niacinamide $(0.3 \%)$ and fenofibrate $(2.4 \%)$. The median number of non-statin antilipemic prescriptions filled per treated individual during follow-up before the use of any statins was 5.0 (interquartile range: 2.0-10.0) with median 242 (interquartile range 97.5-513.0) days of exposure.

\section{Statin Therapy and Subsequent BCC Risk}

A subsequent BCC diagnosed more than 90 days after index date was observed in 6,381 subjects, with $83.4 \%$ occurring during the first five years of follow-up. Using an ever vs. never approach, statin use was associated with a $16 \%$ higher risk of $\mathrm{BCC}(\mathrm{P}=0.004$, Table 1$)$. However, after adjustment for age, gender, and healthcare utilization, the results were no longer statistically significant (adjusted hazard ratio [aHR] 1.02, 95\% CI: 0.90-1.12). A sensitivity analysis which included all subsequent BCCs starting one day after index date demonstrated similar results (aHR 1.07, 95\% CI: 0.95-1.19). Additional sensitivity analyses including only those subsequent BCCs occurring over 180 days and over 365 days after the index date did not alter the results (data not shown). Similarly, sensitivity analysis adding a 90-day lag time before statins would have an impact on BCC formation did not alter the results (aHR 1.00, 95\% CI: 0.90-1.11). 


\section{Cumulative Statin Exposure and Subsequent BCC}

For each additional year of statin exposure, there was no statistically significant change in subsequent BCC risk after adjusting for age, sex, and healthcare utilization (aHR 1.02, 95\% CI: 0.99-1.06). Results were unchanged if the 90-day lag in statin exposure was included (aHR $1.02,95 \%$ CI: 0.98-1.05). Sensitivity analysis that included subsequent BCC occurring anytime after index date showed a $4 \%$ increased adjusted risk of subsequent BCC per year of statin use (aHR 1.04, 95\% CI: 1.00-1.08) that was of borderline statistical significance.

To further clarify the effect of treatment duration on subsequent BCC risk, we categorized cumulative statin exposure. We observed larger point estimates of increased risk for subsequent BCC with increasing duration of exposure to statins compared with no use, but the differences were not statistically significant: short-term use (aHR 0.98, 95\% CI: 0.87-1.11), moderate use (aHR 1.05, 95\% CI: 0.88-1.24) and long-term use (aHR 1.30, 95\% CI: $0.93-1.81, \mathrm{p}=0.20$ for linear trend). Sensitivity analyses that either included all subsequent BCCs occurring anytime after index date or adding a 90 day statin lag did not alter our results (data not shown).

\section{Statin use and BCC risk in treatment-eligible patients}

We repeated all analyses in the subset of individuals who met criteria to receive lipid-lowering therapy based on national clinical practice guidelines incorporating data on low-density lipoprotein cholesterol level and the presence of cardiovascular risk factors ${ }^{20}$ to further limit potential confounding by indication. Of the 1744 subjects included in the analysis, 382 (21.9\%) were lost to follow-up. The mean number of years of follow-up was 4.21 years (median 3.40 years). After adjustment for age, gender and health care healthcare utilization, there was no significant association between statin use or duration of statin use and BCC risk in this treatment-eligible subset (Table 1).

\section{Other lipid-lowering agents and Subsequent BCC}

We also examined the association between use of non-statin antilipemic agents and subsequent BCC. Of the 11932 subjects, 3262 (27.3\%) were lost to follow-up. The mean number of years of follow-up was 4.25 years (median 3.45 years). After adjustment for age, sex and health care utilization, there was no significant relation between any use of non-statin lipid-lowering drugs and subsequent BCC risk (aHR 1.10, 95\% CI: 0.76-1.58).

\section{DISCUSSION}

In a large cohort of individuals at high risk for BCC, we did not observe any significant association between statin exposure and risk of subsequent BCC. The observed crude risk of subsequent BCC was noted to be higher with both any use of statin therapy and with increasing duration of treatment, although these associations were attenuated after adjustment for age, sex and health care utilization. These results were consistent in the overall cohort and in the subset of patients who met national guidelines for eligibility for lipid-lowering therapy. The results were also robust to other sensitivity analyses that assessed different methods of classifying statin exposure (lag 0 vs. 90 days), and identifying subsequent BCCs (arising within 1 day vs. $>90$ days of the index date). In addition, we did not observe any significant association between receipt of non-statin anti-lipemic agents and risk of subsequent BCC, although those analyses had limited power given the infrequent use of these agents in our population.

Studying the epidemiology of non-melanoma skin cancer is difficult because unlike most malignancies, they are not systematically reported to centralized cancer registries. A unique strength of our study was the leveraging of data within a large integrated health care delivery system that included electronic capture of all pathology reports, making it feasible to study the epidemiology of non-melanoma skin cancers. The automated health plan pharmacy database 
facilitated accurate quantification of longitudinal exposure to statin therapy over time, minimizing recall bias. Our follow-up period of up to ten years also allowed assessment of potential long-term effects of statin use on cancer risk. Importantly, our study design focused on a cohort of patients who were previously diagnosed with BCC before the widespread use of statins. This approach mitigates the lack of systematic data on risk factors that contribute to the initial development of BCC such as sun sensitivity, skin type, and history of sun exposure. Finally, we conducted multiple sensitivity analyses that varied cohort and statin exposure definitions, all of which yielded consistent results.

Our findings are consistent with clinical outcome data from several large randomized placebocontrolled trials of statin use for cardiovascular endpoints which observed a slightly increased risk of non-melanoma skin cancer with statin therapy, $21-24$ although the results did not reach statistical significance. In contrast, a recently published article analyzing cancer outcome data from three statin trials did not observe any increased risk of skin cancer. ${ }^{25}$ These trials were designed to assess cardiovascular endpoints rather than non-melanoma skin cancer, which were not systematically ascertained, and the trials were likely underpowered to detect a possible association with statin use.

Selected lines of evidence support the hypothesis that statins may increase the risk of BCC. Statins have been shown to enhance UV phototoxicity in human skin cell lines, an effect that was reversed with cholesterol supplementation. ${ }^{26}$ Certain statins have been associated with UVB phototoxicity. ${ }^{27}$ Also, Smith-Lemli-Opitz syndrome which is characterized by a mutation in the enzyme involved in the last step of cholesterol biosynthesis is associated with marked UVA photosensitivity. ${ }^{28}$ Alternatively, statins could exert possible carcinogenic effects by inhibiting lymphocyte function, ${ }^{29}$ thereby altering immune surveillance. In rodents, statins have also been shown to cause cancer in selected studies at exposure levels comparable with those used in humans. 30

Our study had several limitations. We relied on information from filled prescriptions to characterize statin exposure and were unable to directly measure drug use, which may lead to misclassification. Cohort members may have filled their prescriptions for statins outside a health plan pharmacy, although use of non-Kaiser pharmacies by health plan members is estimated to be rare. ${ }^{31}$ We were not able to examine possible differences between individual statins given that the majority of treated patients received lovastatin. Information about sun exposure history was unavailable, although we attempted to minimize the impact of this by selecting a cohort of patients who by virtue of having one BCC in 1997, shared common risk factor profiles that would make them at similarly increased risk for subsequent BCC. We attempted to control for possible screening bias by adjusting for overall healthcare utilization. We also accounted for confounding by indication by performing a secondary analysis among the subset of patients who appeared eligible for statin therapy based on national clinical practice guidelines.

As an observational study of clinical practice, we cannot completely exclude residual confounding or other treatment selection biases as an alternative explanation of our findings. It is also possible that a small number of subsequent BCCs were treated outside of health plan facilities that were not captured by our pathology database. However, since cohort members already had at least one BCC treated at health plan facilities, it is unlikely that subsequent BCCs would be treated outside the health plan or that this would differ by statin treatment status. Finally, as our study was conducted among insured adults in Northern California, our results may not be completely generalizable to uninsured persons and other health care or geographic settings. 
In conclusion, we found that statin exposure was not associated with a lower risk of subsequent BCC. Point estimates were suggestive of potential higher risk with statin therapy although not statistically significant. Our study detects no association between use of statins, the most commonly prescribed medications in the U.S., and risk of subsequent BCC, the most common cancer. 1,7 Given the ubiquitous and growing use of statin therapy and the public health relevance of BCC, additional studies are needed to clarify the relationship of statins to these cancers.

\section{Acknowledgments}

Funding Sources: This work was supported in part by the National Institute of Arthritis Musculoskeletal and Skin Diseases [K23 AR 051037 to MA, K24 AR 052667 to MC] and by the National Cancer Institute [R01 CA 098838 to $\mathrm{GF}]$.

\section{References}

1. American Cancer Society. Cancer facts and figures 2008. Atlanta: American Cancer Society; 2008 [September 16, 2008]. Available at http://www.cancer.org/downloads/STT/2008CAFFfinalsecured.pdf

2. Miller DL, Weinstock MA. Nonmelanoma skin cancer in the United States: incidence. J Am Acad Dermatol 1994;30:774-8. [PubMed: 8176018]

3. Christenson LJ, Borrowman TA, Vachon CM, Tollefson MM, Otley CC, Weaver AL, Roenigk RK. Incidence of basal cell and squamous cell carcinomas in a population younger than 40 years. JAMA 2005;294:681-90. [PubMed: 16091570]

4. Karagas MR, Stukel TA, Greenberg ER, Baron JA, Mott LA, Stern RS. Risk of subsequent basal cell carcinoma and squamous cell carcinoma of the skin among patients with prior skin cancer. Skin Cancer Prevention Study Group. JAMA 1992;267:3305-10. [PubMed: 1597912]

5. Housman TS, Feldman SR, Williford PM, Fleischer AB Jr, Goldman ND, Acostamadiedo JM, et al. Skin cancer is among the most costly of all cancers to treat for the Medicare population. $\mathrm{J}$ Am Acad Dermatol 2003;48:425-9. [PubMed: 12637924]

6. Marcil I, Stern RS. Risk of developing a subsequent nonmelanoma skin cancer in patients with a history of nonmelanoma skin cancer: a critical review of the literature and meta-analysis. Arch Dermatol 2000;136:1524-30. [PubMed: 11115165]

7. The top 200 prescriptions for 2008 by number of US prescriptions dispensed. [September 16, 2008]. at http://www.rxlist.com/script/main/hp.asp

8. Gniadecki R. Depletion of membrane cholesterol causes ligand-independent activation of Fas and apoptosis. Biochem Biophys Res Commun 2004;320:165-9. [PubMed: 15207716]

9. Cooper MK, Wassif CA, Krakowiak PA, Taipale J, Gong R, Kelley RI, et al. A defective response to Hedgehog signaling in disorders of cholesterol biosynthesis. Nat Genet 2003;33:508-13. [PubMed: 12652302]

10. Corcoran RB, Scott MP. Oxysterols stimulate Sonic hedgehog signal transduction and proliferation of medulloblastoma cells. Proc Natl Acad Sci U S A 2006;103:8408-13. [PubMed: 16707575]

11. Tang JY, So PL, Epstein EH Jr. Novel Hedgehog pathway targets against basal cell carcinoma. Toxicol Appl Pharmacol 2007;224:257-64. [PubMed: 17276471]

12. Chan KK, Oza AM, Siu LL. The statins as anticancer agents. Clin Cancer Res 2003;9:10-9. [PubMed: 12538446]

13. Khan SG, Saxena R, Bickers DR, Mukhtar H, Agarwal R. Inhibition of ras p21 membrane localization and modulation of protein kinase $\mathrm{C}$ isozyme expression during regression of chemical carcinogeninduced murine skin tumors by lovastatin. Mol Carcinog 1995;12:205-12. [PubMed: 7727042]

14. Friedman GD, Flick ED, Udaltsova N, Chan Pharm DJ, Quesenberry CP Jr, Habel LA. Screening statins for possible carcinogenic risk: up to 9 years of follow-up of 361859 recipients. Pharmacoepidemiol Drug Saf 2008;17:27-36. [PubMed: 17944002] 
15. Farwell WR, Scranton RE, Lawler EV, Lew RA, Brophy MT, Fiore LD, et al. The Association Between Statins and Cancer Incidence in a Veterans Population. J Natl Cancer Inst 2008;100:1349. [PubMed: 18182618]

16. Ford I, Murray H, Packard CJ, Shepherd J, Macfarlane PW, Cobbe SM. West of Scotland Coronary Prevention Study Group. Long-term follow-up of the West of Scotland Coronary Prevention Study. N Engl J Med 2007;357:1477-86. [PubMed: 17928595]

17. Bonovas S, Filioussi K, Tsavaris N, Sitaras NM. Statins and cancer risk: a literature-based metaanalysis and meta-regression analysis of 35 randomized controlled trials. J Clin Oncol 2006;24:480817. [PubMed: 17001070]

18. Go AS, Lee WY, Yang J, Lo JC, Gurwitz JH. Statin therapy and risks for death and hospitalization in chronic heart failure. JAMA 2006;296:2105-11. [PubMed: 17077375]

19. Suissa S. Immortal time bias in pharmaco-epidemiology. Am J Epidemiol 2008;167:492-9. [PubMed: 18056625]

20. Executive Summary of the Third Report of the National Cholesterol Education Program (NCEP) Expert Panel on Detection, Evaluation, and Treatment of High Blood Cholesterol in Adults (Adult Treatment Panel III). JAMA 2001;285:2486-2497. [PubMed: 11368702]

21. Downs JR, Clearfield M, Weis S, Whitney E, Shapiro DR, Beere PA, et al. Primary prevention of acute coronary events with lovastatin in men and women with average cholesterol levels: results of AFCAPS/TexCAPS. Air Force/Texas Coronary Atherosclerosis Prevention Study. JAMA 1998;279:1615-22. [PubMed: 9613910]

22. Pfeffer MA, Keech A, Sacks FM, Cobbe SM, Tonkin A, Byington RP, et al. Safety and tolerability of pravastatin in long-term clinical trials: prospective Pravastatin Pooling (PPP) Project. Circulation 2002;105:2341-6. [PubMed: 12021218]

23. Bjerre LM, LeLorier J. Do statins cause cancer? A meta-analysis of large randomized clinical trials. Am J Med 2001;110:716-23. [PubMed: 11403756]

24. Rossebø AB, Pedersen TR, Boman K, Brudi P, Chambers JB, Egstrup K, et al. Intensive lipid lowering with simvastatin and ezetimibe in aortic stenosis. N Engl J Med 2008;359:1343-56. [PubMed: 18765433]

25. Peto R, Emberson J, Landray M, Baigent C, Collins R, Clare R, et al. Analyses of Cancer Data from Three Ezetimibe Trials. N Engl J Med 2008;359

26. Quiec D, Mazière C, Auclair M, Santus R, Gardette J, Redziniak G, et al. Lovastatin enhances the photocytotoxicity of UVA radiation towards cultured N.C.T.C. 2544 human keratinocytes: prevention by cholesterol supplementation and by a cathepsin inhibitor. Biochem J 1995;310:3059. [PubMed: 7646460]

27. Marguery MC, Chouini-Lalanne N, Drugeon C, Gadroy A, Bayle P, Journe F, et al. UV-B phototoxic effects induced by atorvastatin. Arch Dermatol 2006;142:1082-4. [PubMed: 16924075]

28. Chignell CF, Kukielczak BM, Sik RH, Bilski PJ, He YY. Ultraviolet A sensitivity in Smith-LemliOpitz syndrome: Possible involvement of cholesta-5,7,9(11)-trien-3 beta-ol. Free Radic Biol Med 2006;41:339-46. [PubMed: 16814115]

29. Hillyard DZ, Cameron AJ, McDonald KJ, Thomson J, MacIntyre A, Shiels PG, et al. Simvastatin inhibits lymphocyte function in normal subjects and patients with cardiovascular disease. Atherosclerosis 2004;175:305-13. [PubMed: 15262187]

30. Newman TB, Hulley SB. Carcinogenicity of lipid-lowering drugs. JAMA 1996;275:55-60. [PubMed: 8531288]

31. Moffet HH, Adler N, Schillinger D, Ahmed AT, Laraia B, Selby JV, Neugebauer R, Liu JY, Parker MM, Warton M, Karter AJ. Cohort Profile: The Diabetes Study of Northern California (DISTANCE) -objectives and design of a survey follow-up study of social health disparities in a managed care population. Int J Epidemiol. 2008in press 


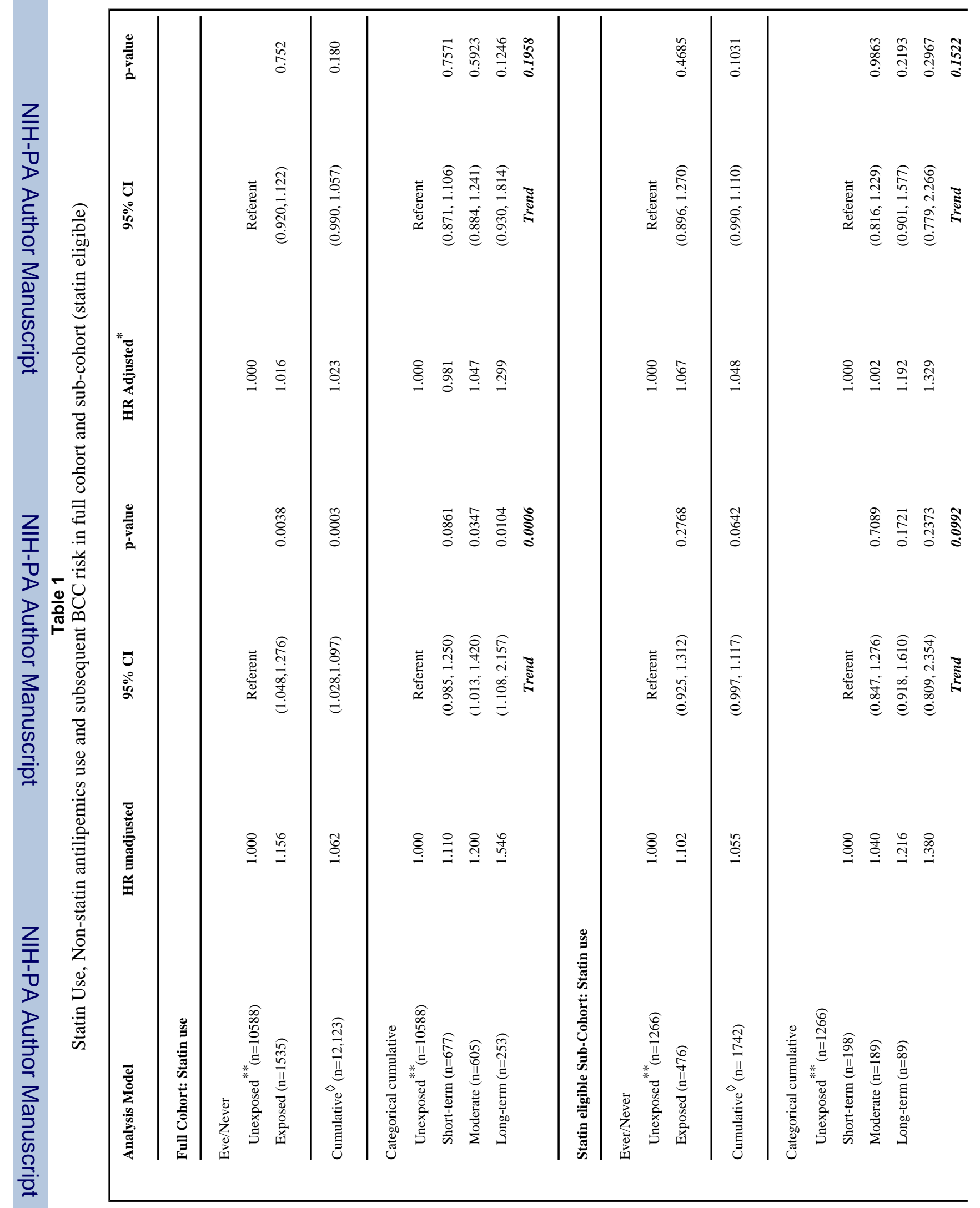

J Am Acad Dermatol. Author manuscript; available in PMC 2010 July 1. 


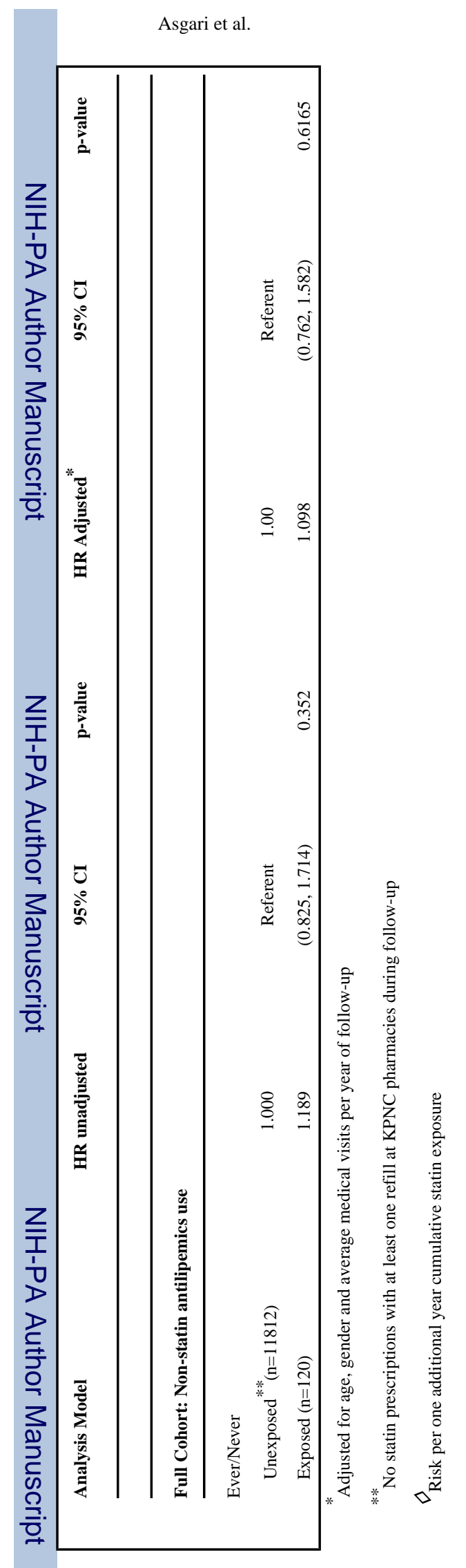

Page 10 